Published in final edited form as:

AIDS. 2012 November 28; 26(18): 2399-2403. doi:10.1097/QAD.0b013e328359aa68.

\title{
Differences between self-reported and electronically monitored adherence among patients receiving antiretroviral therapy in a resource-limited setting
}

\author{
Harsha Thirumurthy ${ }^{a}$, Nalyn Siripong ${ }^{a}$, Rachel C. Vreeman ${ }^{b}$, Cristian Pop-Eleches $^{c}$, James \\ P. Habyarimana ${ }^{d}$, John E. Sidle ${ }^{e}$, Abraham M. Siika $^{f}$, and David R. Bangsberg ${ }^{g, h, i}$ \\ aDepartment of Health Policy and Management, Gillings School of Global Public Health, \\ University of North Carolina at Chapel Hill, Chapel Hill, North Carolina, USA \\ bDepartment of Pediatrics, Indiana University School of Medicine, Indianapolis, Indiana \\ 'School of International and Public Affairs, Columbia University, New York, New York \\ dGeorgetown Public Policy Institute, Georgetown University, Washington, DC \\ eIndiana University School of Medicine, Indianapolis, Indiana, USA \\ 'Moi University School of Medicine, Eldoret, Kenya \\ 9MGH Center for Global Health, Boston, Massachusetts, USA \\ hHarvard Medical School, Boston, Massachusetts, USA \\ 'Ragon Institute of MGH, MIT and Harvard, Boston, Massachusetts, USA
}

\begin{abstract}
Background-Measurement of adherence to antiretroviral therapy (ART) by patient self-report is common in resource-limited settings but widely believed to overstate actual adherence. The extent to which these measures overstate adherence has not been examined among a large patient population.

Methods-HIV-infected adult patients in Kenya who initiated ART within the past 3 months were followed for 6 months. Adherence was measured by participants' self-reports of doses missed in the past 7 days during monthly clinic visits and by continuous Medication Event Monitoring System (MEMS) in participants' pill bottles. Seven-day self-reported adherence was compared to 7-day MEMS adherence, 30-day MEMS adherence, and adherence more than $90 \%$ during each of the first 6 months.
\end{abstract}

Results-Self-reported and MEMS adherence measures were linked for 669 participants. Mean 7-day self-reported adherence was $98.7 \%$ and mean 7-day MEMS adherence was $86.0 \%$, a difference of $12.7 \%(P<0.01)$. The difference between the two adherence measures increased over time due to a decline in 7-day MEMS adherence. However, patients with lower MEMS adherence were in fact more likely to self-report missed doses and the difference between self-reported and MEMS adherence was similar for each number of self-reported missed doses. When analysis was

(C) 2012 Wolters Kluwer Health I Lippincott Williams \& Wilkins

Correspondence to Harsha Thirumurthy, Department of Health Policy and Management, Gillings School of Global Public Health, University of North Carolina at Chapel Hill, Campus Box 7411, Chapel Hill, NC 27599-7411, USA. harsha@unc.edu.

Conflicts of interest

There are no conflicts of interest. 
limited to patients who reported rarely or never removing multiple doses at the same time, mean difference was $10.5 \%(P<0.01)$.

Conclusion-There is a sizable and significant difference between self-reported and MEMS adherence. However, a strong relationship between the measures suggests that self-reported adherence is informative for clinical monitoring and program evaluation.

\section{Keywords}

adherence; adherence measurement; antiretroviral therapy; electronic monitoring

\section{Introduction}

Consistent adherence to HIV antiretroviral therapy (ART) is necessary for viral suppression, prevention of drug resistance, and delaying disease progression [1-3]. Although adherence in resource-limited settings (RLSs) is generally sufficient to achieve viral suppression, various structural and behavioral factors can lead to low adherence and treatment failure on longterm therapy [4-8]. Adherence monitoring is necessary to identify individuals needing intervention, especially in the absence of virologic monitoring.

Patients' self-reports of missed doses have been used extensively because they are inexpensive, easy to implement, and can identify patient-specific barriers to adherence [8,9]. Self-reported adherence has seldom been compared to more objective methods for measuring adherence such as electronic monitoring with Medication Event Monitoring System (MEMS) bottle caps. Most studies using MEMS caps have been conducted in North America and have generally found that self-reports overestimate adherence and are only modestly associated with MEMS estimates [1,8,10-12].

We compared 7-day self-reported adherence to several MEMS adherence measures among patients receiving ART in Kenya in order to determine the strength of association and difference between the two measures.

\section{Methods}

Setting

This study relied on data collected as part of a randomized trial of text messages to improve adherence to ART conducted at the Chulaimbo Rural Health Center (CRHC), a governmentrun health facility in Nyanza Province, Kenya where the USAID-Academic Model Providing Access to Healthcare (AMPATH) Partnership provides comprehensive HIV care at no cost to patients [13].

\section{Study population and procedures}

HIV-infected adults initiating ART at CRHC were recruited from June 2007 until August 2008. Patients were eligible for the study if they were older than 18 years and had initiated ART less than 3 months prior to enrollment. As the primary goal of the study was to evaluate the effect of text messages on adherence, patients were informed that they would receive a mobile phone and that some would be randomly selected to receive daily or weekly text messages encouraging ART adherence. The study was approved by the Institutional Research Ethics Committee of Moi University School of Medicine and the Institutional Review Boards of Georgetown University and the University of North Carolina at Chapel Hill. 
Pharmacy staff transferred one of the participants' three antiretroviral medications (lamivudine) to a bottle with a MEMS cap (Aardex Group, Switzerland) that recorded the date and time of each bottle opening. Participants were informed about the function of MEMS caps during the informed consent process, and they were instructed to open the bottles only when ingesting.

Participants returned every month for appointments with AMPATH clinical officers in accordance with standard clinic procedures. Study staff downloaded MEMS data and obtained patients' reports of the number of doses that they missed in the past 7 days.

\section{Adherence measures}

Data from the return visit questionnaires were used to calculate 7-day self-reported adherence at each clinic visit [(1-number of missed doses/14)×100]. The MEMS data were used to calculate three adherence measures for each return visit:

1. Seven-day MEMS adherence, defined as the percentage of prescribed doses taken during the past 7 days $(100 \times$ number of openings in past 7 days/14);

2. Thirty-day MEMS adherence, defined as the percentage of prescribed doses taken during the past 30 days $(100 \times$ number of openings in past 30 days/60).

3. Binary indicator of whether 7-day MEMS adherence exceeded $90 \%$, a standard for excellent adherence that uniformly achieves viral suppression on nonnucleoside reverse transcriptase inhibitor therapy [14].

The number of daily bottle openingswas truncated to 2 in order to avoid overstating of adherence. Adherence data were analyzed over the first 6 months after recruitment.

\section{Statistical analyses}

For each of the first 6 months of the study, a mean comparison $t$-test was performed to determine whether the difference between 7-day self-reported adherence and 7-day or 30day MEMS adherence was significantly different from zero. This test was also conducted to compare adherence measures during the first 6 months combined.

Some participants reported removing multiple doses at the same time, perhaps to take doses at a later time when they would not have the bottle with them. As this can artificially lower MEMS adherence and is considered a limitation of MEMS measures [15], we also examined MEMS adherence among participants who did not report removing multiple doses at the same time.

\section{Results}

A total of 720 patients were enrolled. MEMS data were obtained from 717 participants. Mean duration of follow-up was 6.2 months [interquartile range (IQR) 3-9 months]. During the first 6 months, 2797 return visit questionnaires were completed among 669 participants and matched to MEMS data. Data entry errors were the most common reason for not matching return visit questionnaires to MEMS data. Participants whose self-reported and MEMS data were not matched had similar age, sex, and education as participants whose data were matched.

Among the 669 participants, $69 \%$ were women and the mean age was 36 years. Thirty-one percent were married and $40 \%$ widowed. More than half the participants (56\%) had completed at least primary school and $22 \%$ had completed secondary school. The mean oneway travel time from home to clinic was $1.47 \mathrm{~h}$ (IQR 0.75-2). 


\section{Self-reported number of missed doses and corresponding Medication Event Monitoring System adherence}

Table 1 tabulates the number of self-reported missed doses in the first 6 months of the study and the corresponding MEMS adherence. In 2541 (90.8\%) return visit interviews, participants reported 0 missed doses during the past 7 days. The mean 7-day MEMS adherence corresponding to each number of self-reported missed doses indicates that participants who reported greater numbers of missed doses also had lower MEMS adherence; and MEMS adherence was found to be 12-14\% lower than self-reported adherence. The proportion with 7-day MEMS adherence more than $90 \%$ was also associated with self-reported adherence, as this was lower for those who reported greater numbers of missed doses. Among those who reported no missed doses during the past 7 days, mean 7day MEMS adherence was $87 \%$ and the proportion with MEMS adherence more than $90 \%$ was 0.73 . In comparison, among those who reported one, two, and three missed doses during the past 7 days, mean 7-day MEMS adherence was 79, 74, and 65\% respectively, whereas the proportion with MEMS adherence more than $90 \%$ was $0.51,0.28$, and 0.39 , respectively.

\section{Comparison of self-reported and Medication Event Monitoring System adherence by month of follow-up}

Seven-day self-reported adherence was high in each month, with a mean of $98.7 \%$ over 6 months. MEMS data indicated significantly lower adherence. Mean 7-day MEMS adherence over 6 months was $86.0 \%$, or $12.7 \%$ lower than 7 -day self-reported adherence $(P<0.01)$. The mean difference between the 7-day self-reported and MEMS adherence measures was similar in the study's intervention and control groups (12.6 and 12.9\%, $P^{1 / 40.74) . ~ T h i r t y-d a y ~}$ MEMS adherence was similar to 7-day MEMS adherence and 12.5\% lower than 7-day selfreported adherence $(P<0.01)$. The proportion with adherence more than $90 \%$ was also lower than what would be expected based on self-reported adherence. Sex, age, school attainment, household size, marital status, alcohol use, and disclosure to somebody in the household were not significantly associated with the difference between self-reported and MEMS adherence.

There was a statistically significant difference between self-reported and MEMS adherence in each month of follow-up $(P<0.01)$ that increased over time due to a decline in MEMS adherence. The difference between 7-day self-reported adherence and 7-day MEMS adherence was $9.8 \%$ at the first month of follow-up and rose to $13.4 \%$ at 6 months; a linear trend was statistically significant $(P<0.01)$. The proportion with 7-day MEMS adherence more than $90 \%$ also declined over time, from 0.76 to 0.68 .

Participants reported having removed multiple doses at the same time in 9.9\% (2797) of the return visits. In visits during which multiple dose removal was not reported, the discrepancy between 7-day self-reported adherence and 7-day MEMS adherence was smaller than those reported in Table 2 but remained statistically significant (mean difference $=10.5 \% ; P<0.01$; results not reported).

\section{Discussion}

Our findings show that although patient-reported measures of adherence are consistently higher than MEMS adherence, there is a significant association between the measures. Selfreported adherence exceeded MEMS adherence by $12.7 \%$, but patients with lower MEMS adherence were in fact more likely to self-report missed doses. Importantly, the difference between the two adherence measures was similar across a range of self-reported adherence; this difference did not get larger for patients with low self-reported adherence. Self-reports of having removed multiple doses at the same time were also consistent with the MEMS 
data; the difference between self-reported and MEMS adherence was narrowed to $10.5 \%$ among participants who did not remove multiple doses. These findings suggest that although self-reports are an imperfect measure of adherence, they can convey useful information.

Three other findings also warrant discussion. First, the finding that self-reported adherence exceeds MEMS adherence is consistent with several other studies in both resource-rich and resource-limited settings $[8,9,12,16]$, but the size of the difference has not previously been estimated among a large cohort of patients in sub-Saharan Africa. The difference between the two measures is significantly smaller than the $25-30 \%$ difference reported in resourcerich settings $[9,16]$ and may be related to the high level of adherence observed in this study.

Second, although self-reported adherence showed minimal decline over time, 7-day and 30day MEMS adherence showed larger declines, from 89.7 to $83.8 \%$ between month 1 and 6 in the case of 30-day adherence. The decline in adherence over time has been attributed to factors including pill fatigue, the plateau of health benefits, and accumulating long-term side-effects $[4,6,17]$. Our findings imply that self-reports may not detect these declines. As declines in adherence in the long-term may contribute to virologic failure, there is a need for greater adherence monitoring and adherence support interventions. Finally, the inability to find predictors of the difference between self-reported adherence and the more objective MEMS measure of adherence suggests that it is not easy to identify which patients are likely to overestimate adherence.

Several limitations of this study merit consideration. First, the findings apply to one study site and may not be generalizable to other patient populations. The large sample, however, may make the findings more comparable to other such sites than existing data. Second, we do not compare MEMS adherence to other adherence measures that may be relatively easier to implement and are also common in RLS, such as pharmacy refill [18]. Nonetheless, quantifying the difference between self-reported and MEMS adherence provides a better sense of how to interpret one of the most widely used adherence measures in RLS. A third limitation is that we lacked clinical characteristics and are, therefore, unable to compare them to the adherence measures.

\section{Conclusion}

In conclusion, we find that although self-reported adherence consistently overestimates adherence, it does contain useful adherence information because the overestimation can be quantified over time. Self-reported adherence measures combined with other objective and feasible measures like those based on pharmacy claims data [18] may be a useful strategy for identifying patients in need of additional adherence support.

\section{Acknowledgments}

The authors are grateful to the patients who participated in the study, CRHC staff, and the study interviewers and data entry teams.

The World Bank Research Group provided financial support for this project under contracts 7142349 and 7144565 funded by the Bank Netherlands Partnership Program (BNPP). This research was also supported in part by a grant to the USAID-AMPATH Partnership from the United States Agency for International Development as part of the President's Emergency Plan for AIDS Relief (PEPFAR). H.T. received support from the National Institute of Child Health \& Human Development (K01HD061605-01A1). 


\section{References}

1. Paterson DL, Swindells S, Mohr J, Brester M, Vergis EN, Squier C, et al. Adherence to protease inhibitor therapy and outcomes in patients with HIV infection. Ann Intern Med. 2000; 133:21-30. [PubMed: 10877736]

2. Bangsberg DR, Hecht FM, Charlebois ED, Zolopa AR, Holodniy M, Sheiner L, et al. Adherence to protease inhibitors, HIV-1 viral load, and development of drug resistance in an indigent population. AIDS. 2000; 14:357-366. [PubMed: 10770537]

3. Wood E, Hogg RS, Yip B, Harrigan PR, O'Shaughnessy MV, Montaner JS. Is there a baseline CD4 cell count that precludes a survival response to modern antiretroviral therapy? AIDS. 2003; 17:711720. [PubMed: 12646794]

4. Ware NC, Idoko J, Kaaya S, Biraro IA, Wyatt MA, Agbaji O, et al. Explaining adherence success in sub-Saharan Africa: an ethnographic study. PLoS Med. 2009; 6:e11. [PubMed: 19175285]

5. Tuller DM, Bangsberg DR, Senkungu J, Ware NC, Emenyonu N, Weiser SD. Transportation costs impede sustained adherence and access to HAART in a clinic population in southwestern Uganda: a qualitative study. AIDS Behav. 2010; 14:778-784. [PubMed: 19283464]

6. Mills EJ, Nachega JB, Bangsberg DR, Singh S, Rachlis B, Wu P, et al. Adherence to HAART: a systematic review of developed and developing nation patient-reported barriers and facilitators. PLoS Med. 2006; 3:e438. [PubMed: 17121449]

7. Ajose O, Mookerjee S, Mills EJ, Boulle A, Ford N. Treatment outcomes of patients on second-line antiretroviral therapy in resource-limited settings: a systematic review and meta-analysis. AIDS. 2012; 26:929-938. [PubMed: 22313953]

8. Mills EJ, Nachega JB, Buchan I, Orbinski J, Attaran A, Singh S, et al. Adherence to antiretroviral therapy in sub-Saharan Africa and North America: a meta-analysis. JAMA. 2006; 296:679-690. [PubMed: 16896111]

9. Liu H, Golin CE, Miller LG, Hays RD, Beck CK, Sanandaji S, et al. A comparison study of multiple measures of adherence to HIV protease inhibitors. Ann Intern Med. 2001; 134:968-977. [PubMed: 11352698]

10. Hinkin CH, Hardy DJ, Mason KI, Castellon SA, Durvasula RS, Lam MN, et al. Medication adherence in HIV-infected adults: effect of patient age, cognitive status, and substance abuse. AIDS. 2004; 18(Suppl 1):S19-25. [PubMed: 15075494]

11. Levine AJ, Hinkin CH, Castellon SA, Mason KI, Lam MN, Perkins A, et al. Variations in patterns of highly active antiretroviral therapy (HAART) adherence. AIDS Behav. 2005; 9:355-362. [PubMed: 16088365]

12. Oyugi JH, Byakika-Tusiime J, Charlebois ED, Kityo C, Mugerwa R, Mugyenyi P, et al. Multiple validated measures of adherence indicate high levels of adherence to generic HIV antiretroviral therapy in a resource-limited setting. J Acquir Immune Defic Syndr. 2004; 36:1100-1102. [PubMed: 15247564]

13. Pop-Eleches C, Thirumurthy H, Habyarimana JP, Zivin JG, Goldstein MP, de Walque D, et al. Mobile phone technologies improve adherence to antiretroviral treatment in a resource-limited setting: a randomized controlled trial of text message reminders. AIDS. 2011; 25:825-834. [PubMed: 21252632]

14. Bangsberg DR. Less than $95 \%$ adherence to nonnucleoside reverse-transcriptase inhibitor therapy can lead to viral suppression. Clin Infect Dis. 2006; 43:939-941. [PubMed: 16941380]

15. Bangsberg D, Hecht F, Charlebois E, Chesney M, Moss A. Comparing objective measures of adherence to HIV antiretroviral therapy: electronic medication monitors and unannounced pill counts. AIDS Behav. 2001; 5:275-281.

16. Arnsten JH, Demas PA, Farzadegan H, Grant RW, Gourevitch MN, Chang CJ, et al. Antiretroviral therapy adherence and viral suppression in HIV-infected drug users: comparison of self-report and electronic monitoring. Clin Infect Dis. 2001; 33:1417-1423. [PubMed: 11550118]

17. Byakika-Tusiime J, Crane J, Oyugi JH, Ragland K, Kawuma A, Musoke P, et al. Longitudinal antiretroviral adherence in HIV+ Ugandan parents and their children initiating HAART in the MTCT-Plus family treatment model: role of depression in declining adherence over time. AIDS Behav. 2009; 13(Suppl 1):82-91. [PubMed: 19301113] 
18. Bisson GP, Gross R, Bellamy S, Chittams J, Hislop M, Regensberg L, et al. Pharmacy refill adherence compared with CD4 count changes for monitoring HIV-infected adults on antiretroviral therapy. PLoS Med. 2008; 5:e109. [PubMed: 18494555] 


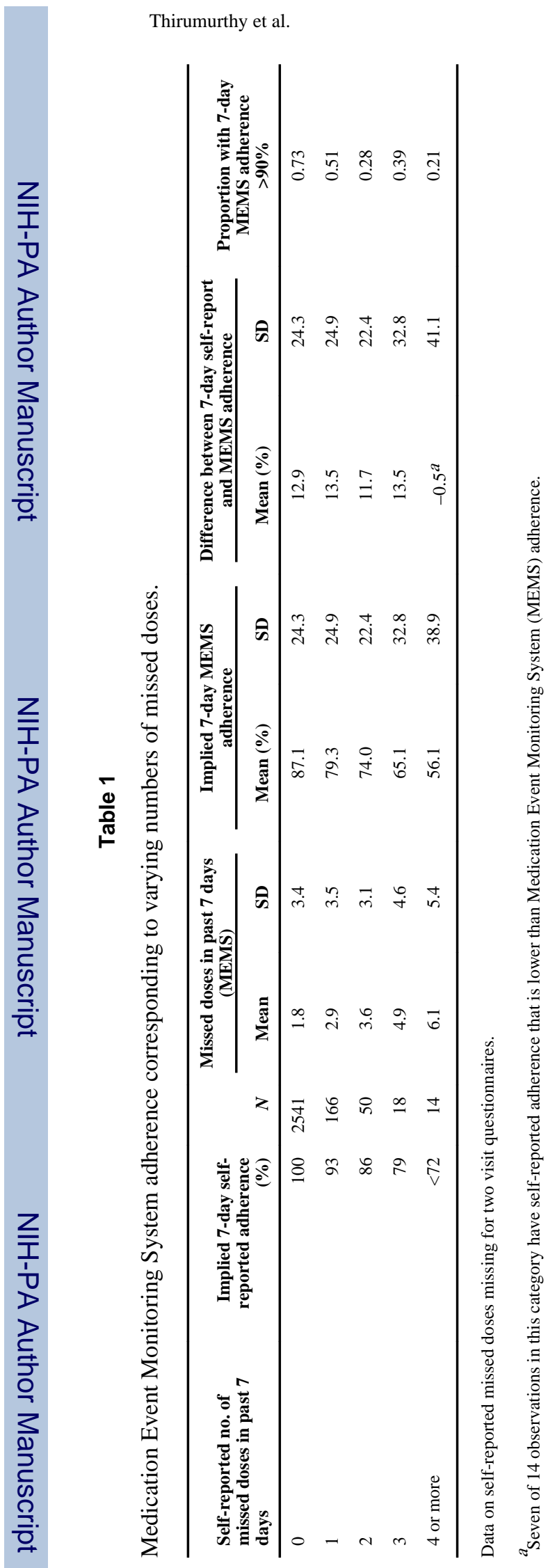

Page 8 


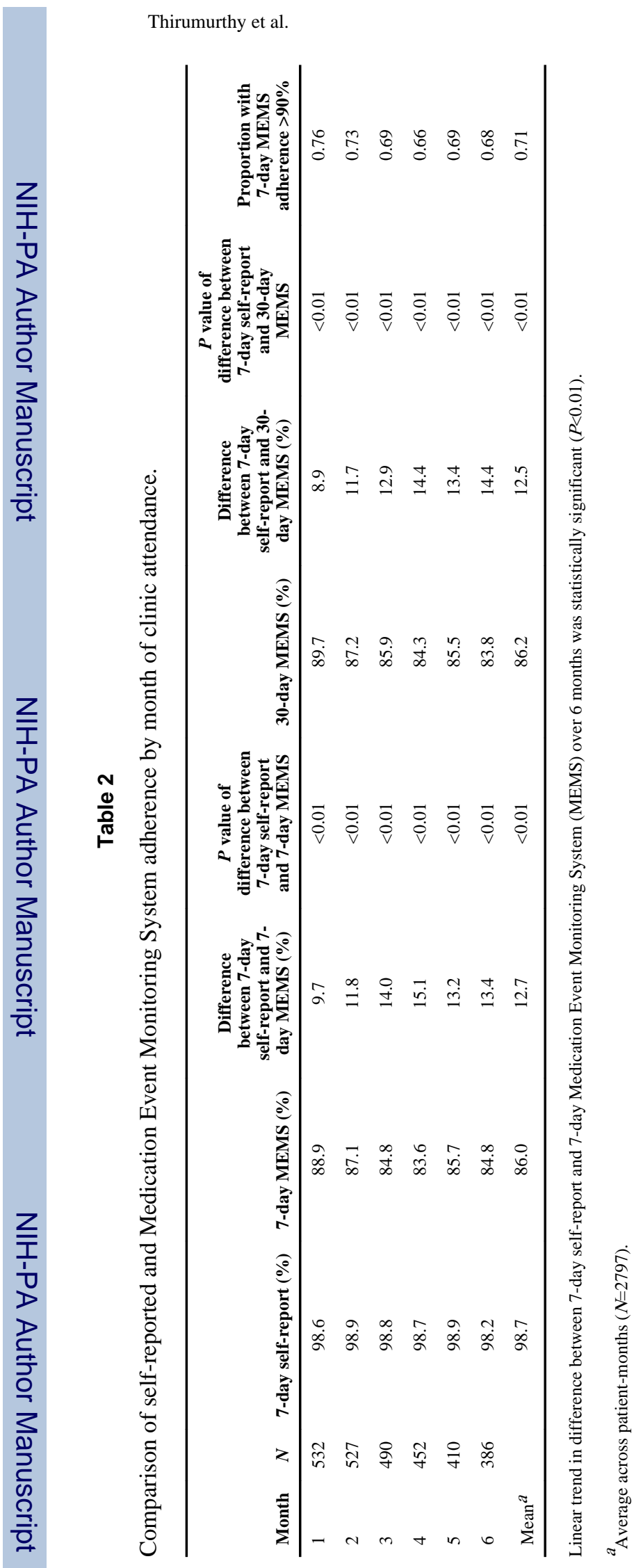

AIDS. Author manuscript; available in PMC 2013 November 28. 